\title{
Symplectic Structures on Integral Manifolds
}

\author{
S. SHAHSHAHANI
}

Communicated by the Editors

Let $\left(M^{2 n}, \omega\right)$ be a symplectic manifold, $H: M \rightarrow \mathbf{R}$ a smooth function, and $X_{H}$ the associated Hamiltonian system, i.e., the unique tangent vectorfield determined by $\left.X_{H}\right\lrcorner \omega=d H$. The Poisson bracket $\{f, g\}$ of two smooth functions $f$ and $g$ is defined by $\{f, g\}=\omega\left(X_{f}, X_{o}\right)$. A first integral for $X_{H}$ is a smooth function $f$ satisfying $\{f, H\}=0$ (equivalently, $f$ is constant on the trajectories of $X_{H}$ ). A set of first integrals $H_{1}=H, H_{2}, \cdots, H_{k}$ for $X_{H}$ which are independent (i.e., $d H_{1}, \cdots, d H_{k}$ are everywhere linearly independent) define a codimension $k$ foliation of $M$ into 'regular integral manifolds'. Moreover, if $H_{1}, \cdots, H_{k}$ are in involution, i.e., $\left\{H_{i}, H_{i}\right\}=0$ for all $i, j=1, \cdots, k$, then all $X_{H_{i}}$ 's are tangent to the regular integral manifolds; it follows that in this case $k \leqq n$. Note, furthermore, that a locally-free action of $\mathbf{R}^{k}$ on a regular integral manifold $I$ is obtained if the vectorfields $X_{H_{i}}$ are complete.

Our main result is:

Theorem. Let $\left(M^{2 n}, \omega\right)$ be a sympletic manifold, $\left\{H_{1}, \cdots, H_{2 r}\right\}$ an even number of functions in involution each generating a complete Hamiltonian vectorfield, and I a regular integral manifold. Then I admits a symplectic structure $\bar{\omega}$ relative to which each $X_{H_{i}}$ is symplectic, i.e., $\left.X_{H_{i}}\right\lrcorner \bar{\omega}$ is closed.

In the case of just two integrals, $H=H_{1}$ and $H_{2}$, for a Hamiltonian system $X_{H}$ the involution property is automatic. Thus for example:

Corollary. In the planar $n$-body problem of Newtonian mechanics, every regular energy-angular momentum level I admits a symplectic structure $\bar{\omega}$ relative to which $X_{H} \mid I$ is a symplectic vectorfield.

It should be pointed out that the incompleteness of the flow in the above example causes no difficulty. Although the forthcoming proof assumes completeness for the sake of elegance, the arguments also hold for a local one-parameter group of diffeomorphisms. The energy-angular momentum levels of the $n$-body problem have been explicitly described by Smale [3].

Before proving the theorem, we point out the following generalization of Darboux's theorem: 
Lemma. If $H_{1}, \cdots, H_{k}$ are independent functions in involution on $\left(M^{2 n}, \omega\right)$ and $x \& M$, there are local coordinates $\left(H_{1}, \cdots, H_{k}, H_{k+1}, \cdots, H_{n} ; x_{1}, \cdots, x_{n}\right)$ around $x$ so that $\omega=\sum d H_{i} \wedge d x_{i}$ and $X_{H_{i}}=\partial / \partial x_{i}, i \leqq k$.

A proof of the above may be obtained by combining Darboux's theorem, Hamiltonian flow-box theorem [1], and an induction [2].

Proof of the theorem. Let $\omega_{1}$ be the two-form on $I$ defined by $\omega_{1}=\omega \mid I$. As $H_{1}, \cdots, H_{2 r}$ determine the integral manifold, it follows from the above lemma that a local expression for $\omega_{1}$ is $\sum_{i>2 r} d H_{i} \wedge d x_{i}$, and hence the rank of $\omega_{1}$ is $2 n-4 r$. We define a second two-form $\omega_{2}$ on $I$ as follows. Regarding the commuting complete vectorfields $X_{H_{1}}, \cdots, X_{H_{2 r}}$ as an action $\mathrm{R} \times I \rightarrow I$, let $L$ be an orbit of the action. Since this action on $L$ is transitive and locally-free, we may in fact regard it as an action $A: F \times L \rightarrow L$, where $F \approx \mathbf{R}^{s} \times T^{2 r-s}$, $0 \leqq s \leqq 2 r$, is the quotient group of $\mathbf{R}^{2 r}$ by the discrete stabilizer of a point in $L$. Note also that $F$ inherits a translation-invariant symplectic form $\omega_{0}$ from the standard symplectic form on $\mathbf{R}^{2 r}$. For each $x \varepsilon L$, the diffeomorphism $\varphi_{x}: F \rightarrow L$ defined by $\varphi_{x}(g)=A(g, x)$ then induces a symplectic 2-form $\left(\omega_{2}\right)_{L}$ on $L$. We show that $\left(\omega_{2}\right)_{L}$ is independent of $x \varepsilon L$. If $y \varepsilon L$ and $\varphi_{y}$ is the corresponding diffeomorphism, a unique element $h \varepsilon F$ is defined by $\left(\varphi_{y}^{-1} \circ \varphi_{x}\right)(0)=h$, or equivalently, $x=\varphi_{y}(h)$. Now if $g$ is an arbitrary element of $F, \varphi_{x}(g)=A(g, x)=$ $A\left(g, \varphi_{y}(h)\right)=A(g, A(h, y))=A(g+h, y)$, or $\left(\varphi_{y}^{-1} \circ \varphi_{x}\right)(g)=g+h$. Therefore $\varphi_{y}^{-1} \circ \varphi_{x}: F \rightarrow F$, being a translation, leaves $\omega_{0}$ invariant, and hence $\left(\omega_{2}\right)_{L}$ is independent of $x \varepsilon L$.

The two-form $\omega_{2}$ on $I$ is then defined via its restrictions $\left(\omega_{2}\right)_{L}$ to the disjoint orbits $L$. The smoothness of $\omega_{2}$ follows from the smoothness of the action of $\mathbf{R}^{2 r}$ and the resulting foliation into orbits.

We next show that $\bar{\omega}=\omega_{1}+\omega_{2}$ is the desired symplectic form on $I$. That $\bar{\omega}$ is closed is trivial. The ranks of $\omega_{1}$ and $\omega_{2}$ are respectively $2 r$ and $2 n-4 r$ which add up to the dimension of $I$. It then suffices to show that the kernels of the maps $X \mapsto i_{X} \omega_{1}$ and $X \mapsto i_{X} \omega_{2}$ are disjoint. The local expression $\omega_{1}=\sum_{i>2 r} d H_{i}$ $\wedge d x_{i}$ shows that the kernel of the first map is in the direction of the orbits of the action $\mathbf{R}^{2 r} \times I \rightarrow I$, i.e., in the direction determined by $x_{1}, \cdots, x_{2 r}$. But $\omega_{2}$ is of maximal rank on the orbits, therefore the claim is proved.

Finally, to show each $X_{H_{i}}, i=1, \cdots, 2 r$, is a symplectic vectorfield on $\underline{A}(I, \bar{\omega})$, first note that $\mathrm{L}_{\left.X_{H(i}\right)} \omega_{1}=0$ by the local expression of $\omega_{1}$, where $H(i)=$ $H_{i}$. Further, if $x \varepsilon I$ and $L$ is the orbit through $x$, the symplectic diffeomorphism $\varphi_{x}^{-1}:\left(L,\left(\omega_{2}\right)_{L}\right) \rightarrow\left(F, \omega_{0}\right)$ takes the flow of $X_{H_{i}}$ to a (symplectic) translation flow on $F$, and hence $L_{X_{H(i}} \omega_{2}=0$. This finishes the proof.

\section{Remarks.}

(1) Let $I$ be an integral manifold as in the theorem, and consider the equivalence relation ' $\sim$ ' on $I$ of belonging to the same orbit of the action of $\mathrm{R}^{2 r}$. The quotient space $\tilde{I}=I / \sim$, known as the 'reduced integral manifold', sometimes admits a natural manifold structure with the projection $p: I \rightarrow \tilde{I}$ a smooth locally trivial submersion. In this case the above proof has a more geometric 
interpretation. By Diff $(F, \Omega)$ we shall mean the group of symplectic diffeomorphisms of a symplectic manifold $(F, \Omega)$.

Lemma. Let $p: E \rightarrow B$ be a smooth fiber bundle with fiber $F$ and structural group $G$. If $B$ and $F$ admit symplectic structures $\omega_{1}$ and $\omega_{2}$ respectively, and if $G \subset \operatorname{Diff}\left(F, \omega_{2}\right)$, then $E$ admits a natural symplectic structure.

Proof. For each $x \varepsilon B$, let $E_{x}$ be the fiber over $x$. As $G \subset \operatorname{Diff}\left(F, \omega_{2}\right)$, we may choose diffeomorphisms $\varphi_{x}: E_{x} \rightarrow F$ so that for all $x, y \varepsilon B,\left(\varphi_{y} \circ \varphi_{x}^{-1}\right)^{*} \omega_{2}=\omega_{2}$. $\varphi_{x}$ then gives rise to a well-defined symplectic form on $E_{x}$ that varies smoothly with $x$. We then obtain a smooth two-form $\omega_{2}^{\prime}$ on $E$. It is easy to check that $\omega=\omega_{2}^{\prime}+p^{*} \omega_{1}$ is symplectic on $E$.

Now consider the case where $p: I \rightarrow I$ is a smooth fiber bundle, and assume without the loss of generality that $\tilde{I}$ is connected. The first lemma of this paper shows that $\left(H_{2 r+1}, \cdots, H_{n} ; x_{2 r+1}, \cdots, x_{n}\right)$ is a set of local coordinates for $\tilde{I}$, and $\tilde{I}$ admits a symplectic form $\sum_{i>2 r} d H_{i} \wedge d x_{i}=\omega_{1}$. Moreover, our proof that $\left(\omega_{2}\right)_{L}$ is independent of $x \varepsilon L$ (see the proof of the theorem) can be interpreted as the reduction of the structural group of $p: I \rightarrow \tilde{I}$ to the group of (symplectic) translations of the fiber. Thus the resulting form $\bar{\omega}$ agrees with that given by the above lemma.

(2) Still in the above setting, if the integrals $H_{1}, \cdots, H_{2 r}$ arise from an action of the Lie group $F=\mathbf{R}^{s} \times T^{2 r-s}, 0 \leqq s \leqq 2 r$, and if the orbits are homeomorphic to $F$, the proof actually shows that $p: I \rightarrow \tilde{I}$ has the structure of a principal fiber bundle. In particular for $s=2 r$, the fiber type is a solid space [4] and hence $p: I \rightarrow \tilde{I}$ admits a global section making it a trivial principal bundle.

(3) The methods above yield other results of similar nature, e.g., the total space of a smooth orientable two-plane bundle over a symplectic manifold admits a symplectic structure since $G L^{+}(2, \mathbf{R}) / S L(2, \mathbf{R})$ is homeomorphic to the space of positive real numbers which is contractible; hence the structural group can be reduced [4].

(4) One cannot, in general, expect the restriction $X_{H_{i}} \mid I$ to be globally Hamiltonian on $(I, \bar{\omega})$. If $I$ is compact, any smooth function on $I$ has a critical point so that the corresponding Hamiltonian system cannot belong to an independent set of vectorfields. For example, in the case of $2 r$ uncoupled harmonic oscillators with Hamiltonian functions $H_{1}, \cdots, H_{2 r}, I$ is generically a $2 r-$ dimensional torus with $X_{H_{i}} \mid I$ merely locally Hamiltonian.

\section{REFERENCES}

1. R. Abraham, Foundations of mechanics, Benjamin, N. Y. 1967.

2. S. Shahshahani, Lecture notes on mechanics, (to appear).

3. S. Smale, Topology and mechanics II, Inventiones Math. 11 (1970), 45-64.

4. N. Steennod, Topology of fibre bundles, Princeton, 1951.

This work was partially supported by National Science Foundation Grant GP-26287.

University of Winsconsin

Date communicated: SePTEMBER 19, 1972 